\title{
An Emergent Way to Manage Patients
}

Can J Neurol Sci. 2014; 41: 143

We are in an era where medical knowledge is in constant escalation and patients are investigated with more sophisticated tools than ever before. However, it is also an era of budget restrictions in which healthcare policies pressure physicians to have a more efficient and economic way to provide services. Although organizing healthcare is a time consuming effort for the already overwhelmed physicians, it does seem to pay off by increasing control of the way outpatient care is provided, where targeted patients may obtain expedited investigation at the right time and place as shown in Wile DJ, et al. ${ }^{1}$

In this ten year retrospective study, the authors reviewed the advantages of having created an Urgent Neurology Clinic in their catchment population area of close to two million. Over that time frame, 12,460 referrals were accepted and seen on average within 3.8 working days. With $44.6 \%$ of these patients ending up with significant change in their neurological diagnosis, not only was patient management improved but the availability of this clinic also decreased the pressure load on the on-call neurology team with a $35.7 \%$ reduction of emergency room neurological assessments. A very significant reduction of in-hospital admission for neurological investigation of 50\% was also achieved.

In our center (Hôpital de l'Enfant-Jésus du CHU de Québec), another kind of outpatient emergency clinic was created three years ago: "la Clinique d'ICT" - (the Transient Ischemic Attack Clinic), where a different population was referred (a population not directly assessed in the Calgary Study by Wile since their transient ischemic attack patients are already referred to their Stroke Prevention Clinic). During the first three years, 672 patients were referred mostly by neurologist on-call, after discussion with either ER physician or physician in an outpatient clinic, that number nearly doubled between the first and the third year. Patients were assessed on average 1.3 days after their referral. They had same day access to imaging (CT or MRI), carotid Doppler, echocardiogram and Holter. Hospitalization was required in only $7 \%$ of these patients, most of them for carotid revascularisation. Before the existence of this clinic, the majority of those patients required rapid evaluation in emergency room or in-hospital usually lasting three to four days since regular outpatient delays in investigation were judged inappropriate. Patients usually reported positively about their experience as information on risk factors management, nutrition and smoking cessation recommendations are also part of the "kit".

Other examples of specialized neurology clinic reported in the scientific literature are scarce. Some models, like the unique Lariboisière Emergency Headache Center in Paris, France (directed by a neurologist) are quite innovative. ${ }^{2}$ Other ones are nearer to the Calgary model, like at the Royal Prince Alfred Hospital in Sydney, Australia. There, 311 patients were referred over a one year period at the "ED Rapid Access Neurology Clinic". Data analysis demonstrated that this clinic prevented 83 unnecessary admissions and 188 out-of-hours neurology consultations, associated once again with high-level of patient satisfaction. ${ }^{3}$ However, there is still insufficient data about these clinics in the medical literature, probably because they are under reported.

Despite a strong adhesion and will from neurologists to investigate patient in an ambulatory setting, they can hardly achieve this goal alone. Without efficient staffing including nurses and clerical support, it is difficult and time-consuming to organise the investigations and follow up. Since the efficiency of such clinics is proven, organisations should support and facilitate such initiatives. For example, access to imaging and other investigation should be negotiated and guaranteed in an appropriate time frame. It is essential to stay patient-focused and provide adequate support and information through the investigations to avoid having patients feeling disappointed without any resource in favor of a cost-efficient service.

In conclusion, this article gives us the opportunity to rethink the way we can improve the availability of daily outpatient service for specialized neurological care in Canada. Not only would resources be more wisely directed, but we could expect improved patient care in terms of quality of life, morbidity and even mortality. Isn't that the noblest goal we all had when we decided to become health care providers?

Martin Savard, Ariane Mackey, Catherine Balg Département des Sciences Neurologiques, Hôpital de l'EnfantJésus du CHU de Québec, Québec, Canada Email: martinsavard17@hotmail.com

\section{REFERENCES}

1. Wile DJ, Warner J, Murphy W, Lafontaine AL, Hanson A, Furtado S. Referrals, wait times and diagnoses at an urgent neurology clinic over 10 years. Can J Neurol Sci. 2014;41(2):260-4.

2. Valade D. The Emergency Headache Center at the Lariboisière Hospital: 7 years with more than 70,000 patients. Intern Emerg Med. 2008;Suppl 1:S3-7.

3. Ahmed RM, Green T, Halmagyi GM, Lewis SJ. A new model for neurology care in the emergency department. Med J Aust. 2010; 192(1):30-2. 